\title{
Tangence
}

\section{Figures et spectres}

\section{Figures and Ghosts}

\section{Justin Bisanswa}

Numéro 75, été 2004

Les formes transculturelles du roman francophone

URI : https://id.erudit.org/iderudit/010784ar

DOI : https://doi.org/10.7202/010784ar

Aller au sommaire du numéro

\section{Éditeur(s)}

Tangence

\section{ISSN}

1189-4563 (imprimé)

1710-0305 (numérique)

Découvrir la revue

\section{Citer cet article}

Bisanswa, J. (2004). Figures et spectres. Tangence, (75), 63-82. https://doi.org/10.7202/010784ar

\section{Résumé de l'article}

Quel rapport le roman africain entretient-il avec le réalisme ? Afin de mettre en évidence la richesse et la multiplicité des formes romanesques, cet article entend montrer que le rapport mimétique que les écrivains africains croyaient cultiver avec le monde était en partie illusoire et que leur prétention à la transparence masquait les ruses et les procédés d'une rhétorique. Que le roman africain ne soit pas aussi mimétique que le prétend une certaine critique ne l'empêche pas de nous en dire beaucoup sur une réalité toujours ancrée dans l'Histoire et de tenter d'en cerner la vérité. Quand il va jusqu'au bout de ce qui le meut, il est un instrument hors pair d'analyse des rouages et des mécanismes sociaux. Là où le roman africain réussit le mieux à nous dire la vérité du social, c'est à même le romanesque, à même son imaginaire, à même son écriture ou sa poétique.
Ce document est protégé par la loi sur le droit d'auteur. L'utilisation des services d'Érudit (y compris la reproduction) est assujettie à sa politique d'utilisation que vous pouvez consulter en ligne.

https://apropos.erudit.org/fr/usagers/politique-dutilisation/ 


\title{
Figures et spectres ${ }^{1}$
}

\author{
Justin Bisanswa, \\ Université Laval
}

Quel rapport le roman africain entretient-il avec le réalisme? Afin de mettre en évidence la richesse et la multiplicité des formes romanesques, cet article entend montrer que le rapport mimétique que les écrivains africains croyaient cultiver avec le monde était en partie illusoire et que leur prétention à la transparence masquait les ruses et les procédés d'une rhétorique. Que le roman africain ne soit pas aussi mimétique que le prétend une certaine critique ne l'empêche pas de nous en dire beaucoup sur une réalité toujours ancrée dans l'Histoire et de tenter d'en cerner la vérité. Quand il va jusqu'au bout de ce qui le meut, il est un instrument hors pair d'analyse des rouages et des mécanismes sociaux. Là où le roman africain réussit le mieux à nous dire la vérité du social, c'est à même le romanesque, à même son imaginaire, à même son écriture ou sa poétique.

\section{Une autre lecture}

Africain ou occidentalisé? Telle est la question que se posent certains critiques du texte africain à propos de l'auteur. Dans son implicite, la question soulève la problématique des sources d'inspiration, voire d'écriture, des «formes» du roman africain. Aussi, Josias Semujanga invite-t-il à une «analyse transculturelle» des littératures francophones, qui consiste, selon lui, à examiner comment des modèles "puisés dans telle ou telle culture» sont «retravaillés et fusionnés de façon à ce que soient effacées les traces dans la culture nouvelle qui, tel un limon dans une vallée, est indépendante des matériaux ayant servi à sa formation ${ }^{2} »$. Comment

1. Je remercie le Programme des Chaires de recherche du Canada et le Conseil de recherche en sciences humaines (CRSH) pour leur soutien qui m'a permis de réaliser cette recherche. Je sais également gré au Wissenschaftskolleg de Berlin pour les conditions de travail magnifiques qu'il m'a offertes afin de faire avancer mes projets de recherche.

2. Voir, dans ce numéro, l'article de Josias Semujanga, «La mémoire transculturelle comme fondement du sujet africain chez Mudimbe et Ngal», p. 15-39. 
entendre cette problématique ou la manière de la poser? La phrase orale de Céline, par exemple, n'est pas une influence des littératures orales africaines. Les formalistes russes avaient déjà analysé, sous le nom de $s k a z$, ce mélange de l'oral et de l'écrit, du poétique et du profane, du conventionnel et du non-conventionnel ${ }^{3}$. Cet assemblage de deux codes différents, dont l'un enrichit l'autre, rend le discours textuel dans son ensemble plus dynamique et invite à la participation sur le plan de la communication. Le discours de Vladimir dans En attendant Godot, la scène fantasmatique du viol dans La nausée ou la troisième conversation dans Moderato cantabile font basculer le discours dans le verbal, comme par un effet de syncope ou de télescopage. La critique, il est vrai, veut dresser un bilan des pratiques: au risque d'être partiale, elle veut classifier, catégoriser, étiqueter ${ }^{4}$. Dans Michelet par lui-même, Roland Barthes montrait déjà que l'Histoire est pure forme, et c'est à partir de la forme que je lis souvent l'Histoire dans le roman africain.

Pour varier sur la thématique du numéro, mais de manière à la renouveler en profondeur afin de libérer la lecture des clichés et stéréotypes, je voudrais interroger les "petits trucs» de fabrication du roman africain, c'est-à-dire analyser certains dispositifs narratifs bien agencés et montrer comment la poétique et l'imaginaire disent le social. C'est d'abord, comme l'avait déjà vu Céline, que la société se dérobe à la prise, qu'elle manifeste une complexité et une opacité qui découragent par avance toute entreprise de description totalisante. C'est, en second lieu, que la fiction ne peut plus ignorer comme jadis ce qui fait sa définition essentielle et qui conjugue étroitement création d'un imaginaire et production du langage. Il est vrai que subsiste toute une critique qui fait comme si rien ne s'était passé et qui, à force de banaliser les recettes du réalismenaturalisme africain, a transformé ce dernier en un «naturel» qui est le refus même de l'art. Clairement, cette critique ne participe plus depuis longtemps au processus de création.

«Figures et spectres»! J'aimerais interroger quelques romans africains en relation avec le réalisme, que je préfère appeler le réel pour montrer la richesse et la multiplicité des formes romanesques africaines. Figures et spectres! Je me dis qu'au fond, il y a peut-être

3. Voir Théorie de la littérature, textes des Formalistes russes réunis, présentés et traduits par Tzvetan Todorov, Paris, Seuil, coll. «Tel Quel», 1966, p. 121.

4. Voir Justin Bisanswa, "L'aventure du discours critique africain », Présence francophone, Sherbrooke, $n^{\circ}$ 61, 2003, p. 11-34. 
deux grandes manières seulement, deux grandeurs plutôt, dans cette folie de l'écriture africaine, comme dans n'importe laquelle, qui fait que quiconque écrit s'efface tout en laissant, pour l'abandonner, l'archive de son propre effacement. Deux mesures pour cet acte d'écriture par lequel quiconque écrit feint de s'effacer en nous laissant pris dans son archive comme dans une toile d'araignée.

Je vais simplifier outrageusement. Il y a d'abord la grandeur de qui écrit pour donner, et donc pour donner à oublier le donné et la donnée, ce qui est donné et l'acte de donner. Au-delà de tout retour, de toute circulation, de toute circonférence. C'est la seule manière de donner, la seule possible - et impossible. Avant toute restitution, symbolique ou réelle, avant toute reconnaissance, la simple mémoire, à vrai dire la seule conscience du don, de part ou d'autre, annule l'essence même du don. Celui-ci doit ouvrir ou rompre le cercle, rester sans retour, sans l'esquisse, fût-elle symbolique, d'une reconnaissance. Au-delà de toute conscience, bien sûr, mais aussi de toute structure symbolique de l'inconscient. Le don une fois reçu, l'œuvre ayant fait œuvre jusqu'à vous changer de part en part, la scène est autre et vous avez oublié le don, le donateur ou la donatrice.

L'autre grandeur, je dirai sans le moindre doute qu'elle concerne plutôt l'écriture africaine, les écritures africaines. L'événement s'y déploie dans une intrigue où il prend une envergure telle que, désormais, vous n'avez plus d'autre issue: être en mémoire de lui. Non seulement par lui débordé, mais par lui obligé, contraint de vous mesurer à ce débordement. Être en mémoire de lui: non pas nécessairement vous souvenir de lui, non, être en sa mémoire, habiter une mémoire désormais plus grande que votre souvenir et ce qu'il peut rassembler, en un seul instant ou en un seul vocable, de cultures, langues, mythologies, religions, philosophies, sciences, histoires de l'esprit ou des littératures. D'avance et à jamais, cet acte de guerre babélien, cette hypermnésie vous inscrit dans le roman que vous lisez. C'est là l'amorce de l'intertextualité dont Julia Kristeva a indiqué les deux phases du processus, à savoir l'absorption et l'intégration. Paul Ricœur avait raison de rappeler que «chaque intrigue s'inscrit dans une tradition de l'art de raconter, au sein de laquelle conformité et innovation entrent en concurrence ${ }^{5}$ ». La

5. Paul Ricoeur, Entre herméneutique et sémiotique, Presses de l'Université de Limoges, 1990, p. 12. 
question n'est plus alors de réactiver par compréhension l'acte structurant, mais de décrire selon leur objectivité propre les structures issues de l'opération structurante.

La critique souligne généralement que le roman africain est en prise sur l'Histoire. Néanmoins, une lecture attentive à la forme montre bien que le roman de création ne peut plus se satisfaire du seul modèle réaliste. Entre-temps s'est ouverte «l'ère du soupçon », selon la belle formule de Nathalie Sarraute; elle a voulu que le doute soit jeté sur les fondements du romanesque, depuis l'intrigue jusqu'aux personnages. Elle a induit le genre à se tourner vers lui-même, à cultiver sa forme, à substituer à la référence au monde une référence à soi, c'est-à-dire à la littérature.

On peut noter, du reste, que l'actuelle mauvaise conscience du genre romanesque retentit jusqu'à un certain point sur notre lecture des grands réalistes du passé. On sait désormais que le rapport mimétique que ces derniers croyaient entretenir avec le monde était en partie illusoire et que leur prétention à la transparence masquait les ruses et les procédés d'une rhétorique. Mais on doit admettre aussi que cette notion de réalisme, qui se confond, pour beaucoup, avec celle de l'engagement, est intimement liée à une double histoire, littéraire d'un côté, sociopolitique de l'autre. Elle reste associée à une grande lutte pour la reconnaissance des droits humains et à une grande bataille pour l'art. Si le premier réalisme ne se survit guère, les enjeux les plus essentiels qui y sont rattachés demeurent. Ils concernent une façon de définir le rôle de la littérature en société, de placer celle-ci dans une perspective historique ou même tout simplement de la lire et de la pratiquer.

Que le roman africain ne soit pas aussi mimétique que le prétend la critique ne l'empêche pas de nous en dire beaucoup sur une réalité toujours ancrée dans l'Histoire et de tenter d'en cerner la vérité. Mais qu'on ne s'y méprenne pas. Quand il va jusqu'au bout de ce qui le meut, il est un instrument hors pair d'analyse des rouages et des mécanismes sociaux. Là où le roman africain réussit le mieux à nous dire la vérité du social, c'est à même le romanesque, à même son imaginaire, à même son écriture ou sa poétique. Occasion de rappeler que les romanciers africains sont aussi et peut-être d'abord de grands fabulateurs, de grands conteurs. Ils libèrent l'écriture et donnent à leurs représentations la puissance du symbolique ou la force irradiante du fantasme. Ils restent, toutefois, fidèles à la vocation première du roman en n'atteignant le collectif que par le biais de destins individuels et en 
faisant accéder des personnages quelconques à des rôles héroïques et idéalisés.

Ainsi, forte de ses faux-semblants (dramatiques, pathétiques, psychologiques), la fiction est essentiellement, pour le roman africain, un mode de lecture des complexités sociales. De là, cette observation que l'on peut faire à son propos: ce n'est pas dans ses commentaires sociologisants - mauvaises resucées bien souvent des idéologies du temps —, ni dans ses descriptions de "milieux» trop longtemps tartinées, qu'il dit une vérité sur le monde; c'est là où il invente un univers, là où il dit les rapports humains en des projections qui confinent à l'allégorie, là où il s'approprie les paroles les plus triviales en des artefacts linguistiques, qu'il propose la grille la plus opératoire et la plus perspicace de déchiffrement de la société. Il est donc permis de tenir son entreprise pour un grand laboratoire fictionnel où, au gré de multiples expériences de figuration, l'imaginaire le dispute au «réel». Aussi n'est-il de forme romanesque que dans le dépassement de la forme.

Véritable expérience de totalisation: voilà le roman pensé comme vaste entité organique, qui mime jusqu'au délire la multiplicité et la complexité du monde; le voilà qui enferme l'espace et le temps dans une structure suffisamment vaste pour donner l'impression d'englober toute une société sans craindre de se perdre dans sa représentation. Chaque romancier africain développe évidemment son propre mode de dépassement et nous verrons qu'il n'est pas deux romans qui présentent la même forme. Mais tous sont emportés par la même surenchère qui est d'en dire beaucoup et jusque dans les plus petits détails. Comment s'étonner, dès lors, que les uns se soient déportés jusqu'aux confins du fantastique, que d'autres aboutissent à des visions confinant à l'utopie, que d'autres encore apparentent le romanesque à l'évocation poétique? Les formules abondent pour caractériser les formes du roman africain : réalisme carnavalesque, réalisme fantastique, réalisme satirique, réalisme à thèse, réalisme de combat, réalisme formaliste, hyperréalisme...

Le plus étonnant est que l'objet de pensée et d'action de cette énergie créatrice reste, dans le roman africain, un monde de désenchantement. À l'égal d'un Vigny, d'un Baudelaire ou d'un Apollinaire, les romanciers africains ne peuvent s'empêcher de porter sur le monde le regard mélancolique de ceux qui ont perdu leurs illusions.

Le procès du roman africain et de son interprétation peut donc s'instruire suivant différents angles et s'ouvrir sur le 
questionnement salutaire d'un mode de représentation et d'une esthétique fondés sur des présupposés devenus caducs. On peut d'ailleurs l'inscrire dans le mouvement plus large d'une remise en question par diverses sciences humaines des conditions générales de toute représentation. On sait que la nouvelle poétique a procédé à une véritable table rase des croyances qui soutenaient le vraisemblable mimétique. Le lecteur averti est devenu sensible à ses trucs de fabrication ou, mieux encore, il a appris à lire Ahmadou Kourouma, Sony Labou Tansi ou Calixthe Beyala autrement, en faisant de leurs romans un usage différent. Peu à peu, il s'est prémuni contre la lecture crédule qui confond fiction et réalité et prend au mot le discours du roman. Aucune fiction n'est à prendre pour un document authentique et, moins que toute autre peutêtre, celle-là qui se pose trop facilement comme telle.

Mais, plus avant, nous avons été rendus attentifs au fait que le roman s'affirme pleinement dans une écriture qui, de surcroît, se trouve retorse et dissimulée. Il relève de toutes sortes de conventions expressives. Le style de Sony Labou Tansi est largement métaphorique. Le style oral de Kourouma relève de tout un art de la composition. Quand on y regarde bien, la simplicité d'Ousmane Sembène est un leurre et son discours est truffé de petits indicateurs réalistes. Ajoutons que cette rhétorique charge le texte de valeurs et de fonctions. Par exemple, tout un didactisme, caractéristique des romanciers de cette obédience, consiste à transformer la représentation en savoir à transmettre, et même en leçon. Il peut s'afficher en des excursus plus ou moins savants sur telle ou telle "question », mais peut aussi passer, plus discrètement, par le simple caractère «monstratif» ou démonstratif de l'écriture.

L'effet le plus bénéfique de tout le travail critique opéré a été de libérer la lecture des romanciers africains. Eux que l'on enfermait naguère encore dans une attitude de fidélité aux realia se voient tout à coup reconnaître un droit à la fiction et à l'imaginaire. Les contraintes qu'ils ont imposées à leur propre discours sont en quelque sorte levées au profit d'éléments textuels que les lecteurs, comme eux-mêmes, refoulaient. Plus obscurément, il y a au cour du propos romanesque africain cet acharnement - vrai ou joué, qu'importe - à dire, à voir, à montrer, à savoir. La mimésis est en elle-même hallucination ou folie: elle revient à trouver jouissance dans une création qui se nie dans la mesure où elle ne fait que reproduire, c'est-à-dire produire, ce qui est déjà. Plus que de discréditer le réalisme du roman africain, la prise en compte de l'illusion référentielle ou de l'effet de réel a permis d'en 
faire une lecture plus avisée et, en fin de compte, plus riche. Avec ceci, tout de même: à dénier que les romans de cette obédience parlent du monde, on les prive de toute une part de leur sens historique et de leur portée. Peut-on aller jusqu'à cette poétisation radicale du roman africain? On maintiendra, à ce propos, que le langage parle du monde et qu'il est même fait pour cela. Nous savons qu'il n'en produit pas une simple duplication, mais qu'il en construit la représentation et que cette construction ne va pas sans réfractions diverses. Si le texte littéraire fonctionne en circuit autonome et surimpose son propre système à celui de la langue, il n'en indexe pas moins ses composants sur des référents extérieurs, qu'il le veuille ou non. Le Guide providentiel dans La vie et demie de Sony Labou Tansi n'existe pas dans le réel, mais il a des correspondants dans la réalité: certains dignitaires africains tenants du pouvoir.

La question est de savoir comment le roman africain s'y prend pour connecter ou, mieux encore, homologuer deux univers de sens qui sont tout ensemble très proches et très distincts. Il s'agit soit, à l'intérieur du même texte, d'un simulacre qui tire sa vraisemblance d'une cohésion interne, avec, par ailleurs, l'indexation de ce simulacre sur un univers externe qui est celui de l'Histoire; soit, dans le processus d'écriture-lecture, d'une ambivalence à laquelle chacun est confronté, l'auteur, son lecteur, l'analyste. Tentons de préciser les choses.

\section{Un roman social}

Partons d'un exemple typique. Soit les premiers énoncés du Bel immonde de Valentin-Yves Mudimbe: "Elle attend. Comme chaque soir. Les yeux mi-clos, elle sourit, la main droite caressant paresseusement son châle de soie. Elle espère toujours, cherche son seigneur, visiblement exaltée et lassée à la fois par cette musique envahissante qui l'englue dans une débauche de fumée de cigarettes et des relents d'alcool ${ }^{6} »$. On ne fait guère plus simple, voire plus plat. C'est un effet de réel au sens élargi: il dénote la vie à travers une action déjà commencée et connote la posture d'attente du lecteur à partir de celle du personnage. Mais l'énoncé dit autre chose, et sur deux plans. D'une part, il pointe, fût-ce allusivement,

6. Valentin-Yves Mudimbe, Le bel immonde, Paris, Présence africaine, 1976, p. 17. 
une référence externe socialement fondée, à savoir la prostitution à Kinshasa, à laquelle va se livrer une étudiante pour survivre: voilà une femme en danger d'abandon (désignée par le shifter «Elle» qui l'anonymise), qui guette l'arrivée de son compagnon, désigné parallèlement par un « Il», le pronom de la non-personne, comme disait Barthes. On découvrira que c'est un homme puissant, un ministre. On ne sait rien que, déjà, surgit un contexte de lecture. D’autre part, le même énoncé s'inscrit dans la durée (un avant et un après) d'une relation humaine, dans un drame en gestation. La fille va abandonner ses études, et la vie sociale et politique du ministre va basculer. Ainsi une société prend forme en filigrane, qui n'est plus celle du dehors mais celle, fictive, du dedans: une société du texte. D'un côté, une société réelle posée en horizon connu; de l'autre, une société du texte qui affirme déjà sa cohésion. Elles sont en accord mais aussi distinctes, et telles qu'on ne peut les confondre. C'est dire que la lecture réaliste va se constituer dans leur oscillation réciproque. Ainsi, elle reconnaîtra le contenu de vérité du roman dans la capacité de celui-ci à accorder la société fictive qu'il élabore avec la société réelle à laquelle il fait penser et sur les référents de laquelle, quelle que soit la manière, il s'indexe.

Nous devons en particulier à Claude Duchet d'avoir, sur un mode renouvelé, mis en rapport le réalisme avec la dimension sociale de la pratique humaine ${ }^{7}$. C'est lui qui a défini cette socialité à double face dont se prévaut, sans trop le savoir, toute une tradition romanesque. Ce savoir du discours romanesque aime aussi à prendre les allures d'un contre-savoir, c'est-à-dire à divulguer ce que la doxa interdit de rendre public. Le réalisme du roman africain se fait alors dévoilement toujours plus ou moins scandaleux. Bousculant les tabous, le roman a une vocation d'obscénité, et pas seulement dans l'ordre sexuel. Pour prendre un exemple crucial, il se plaira à exhiber les plaies d'une misère faite pour heurter la bienséance bourgeoise. Décrire la pauvreté dans tous ses détails relève d'une audace scandaleuse qui valut facilement au réalisme du roman africain une réputation de pornographie sociale. De cette misère absolue, Sembène, Kourouma, Lopes et Mudimbe se sont fait une spécialité, dans laquelle les relayera un Sony Labou Tansi volontiers scatologique. Chez d'autres, la misère affichée est davantage d'ordre moral ou psychique. D'être le fait des classes

7. Claude Duchet, «Une écriture de la socialité», Poétique, Paris, n 16, 1973, p. 446-449. 
respectables ne la rend que plus choquante: du Guide providentiel dans La vie et demie au Ministre du Bel immonde, la déchéance des grands de ce monde heurte le bon goût, tout comme peut choquer la bêtise d'un Koyaga dans En attendant le vote des bêtes sauvages, ou l'inaction de Nara dans L'écart.

Dans ce contexte provocateur, le sexuel est l'un des enjeux des textes qui nous occupent. Que le sexe mène le monde, à l'instar de l'argent ou du pouvoir, le roman africain en convient largement ${ }^{8}$. Si les relations sentimentales demeurent pour lui un grand moteur du romanesque, elles trouvent leur nécessaire contrepoint dans l'impatience du désir et de la pulsion charnelle. Ce roman évoquera donc le corps libidinal avec une insistance croissante, mais sans jamais sortir des contradictions inhérentes à sa mise en discours. De sexe masculin, nos romanciers africains ne manquent pas de reconduire une vision de la femme comme objet de désir et proie érotique. Ils prennent aussi conscience du fait que, dans le monde moderne, la femme est appelée à jouer un rôle de plus en plus significatif. Surtout, ils font de maints personnages féminins (Penda, Salimata, Ya, Chaïdana, Yavelde, Moussokoro, etc.) des premiers rôles qu'ils dessinent avec une sensibilité vraie, en leur donnant toute l'ampleur voulue.

La femme est, par ailleurs, prétexte à dire le corps dans sa vérité et sa violence. Mais les mots n'y suffisent guère, et la censure veille. On s'en tire donc par des subterfuges. La "chute» du Ministre et de Ya dans Le bel immonde ainsi que celle du commandant dans Monnè, outrages et défis sont dites de façon détournée et tout allusive, ici par un renvoi à la sensualité de la nature ambiante, là par une mention tout elliptique rappelant la formule de Flaubert à propos d'Emma Bovary: «aucune résistance ne fut opposée». Il n'en émane que plus de trouble. Le roman réaliste ne fait ainsi que se conformer à l'état des mœurs et des convenances. De temps à autre, pourtant, il a ses audaces et risque un regard qui, de Maran à Sony, croît en précision et en impudeur. Sony parle crûment et platement de cette grève des «jambes jointes» dans Les sept solitudes de Lorsa Lopez.

C'est sa manière d'expérimenter les limites du dicible, tout en faisant pièce à l'hypocrisie des mours et des mots. Nommer avec franchise certaines nécessités physiques revient à faire un acte

8. Spécificité africaine, comme le pensent certains critiques? Lire Balzac, Zola, Stendhal, Maupassant, Flaubert, Proust, etc., pour se convaincre du contraire. 
libérateur. C'est un geste qu'accomplit volontiers Sony Labou Tansi, comme lorsqu'il raconte les ébats sexuels du Guide providentiel. On se rappellera Zola évoquant dans Germinal, avec une tranquille franchise, les premières menstrues de Catherine Mahieu après la bataille meurtrière entre grévistes et soldats, ou lorsqu'il donne à voir les femmes de mineurs qui émasculent l'épicier Maigrat dans leur fureur d'exploitées. Ces moments du texte sont d'une vérité juste qui retentit sur tout le roman. Mais, contrairement à Zola qui peut parler des règles et de l'appareil génital des prolétaires qui, selon la doxa, sont encore proches de l'animalité, Sony dit et montre le sexe des puissants dignitaires du régime.

Mais bien que concertée, l'écriture réaliste du roman africain a quelque chose de compulsif. Dans Excès du roman ${ }^{9}$, Tiphaine Samoyaut considère que l'expansion totalisante est une tendance plus générale du roman moderne. Le roman, je l'ai dit, a partie liée avec l'histoire sociopolitique mouvementée de l'Afrique. Tout cela équivaut à un volume énorme d'informations et d'écriture, se diffusant dans le corps social et donnant l'impression d'un flux incessant. Universel reportage où l'information utile finit par se consumer, à force de répétition et de saturation, en une sorte de bruit et de fureur dont de bien plus puissants médias nous procurent bien d'autres exemples aujourd'hui. Inflation scripturale et médiatique qui semble vouloir cribler l'espace de la représentation dans une concurrence effrénée avec un réel lui-même expansionniste et se démultipliant.

Boubacar Boris Diop a collaboré aux journaux. Murambi. Le livre des ossements témoigne de cette immersion dans une pléthore d'écrits qui se veulent la parole instantanée de l'actualité rwandaise. Aussi y a-t-il continuité, pour lui, entre sa pratique littéraire et sa pratique du journalisme. Et c'est sans rupture qu'il s'adonne à une écriture généreuse, proliférante, réceptive au monde comme il va. La forme ne fait pas problème: sans limites et sans règles, le roman est expansif à souhait. Quant au contenu, il suffit de le «journaliser». C'est ce à quoi s'emploie le réalisme d'un Kourouma en prônant, dans Allah n'est pas obligé, des récits proches du fait divers et du quotidien le plus banal: en l'occurrence, le fléau de la guerre dans les pays riches d'Afrique. Et c'est comme si le roman

9. Tiphaine Samoyaut, Excès du roman, Paris, Maurice Nadeau, 1999. L'entreprise réaliste est faite de tout un excès, qui voue le genre romanesque, en tout et en détail, à une expansion infinie. 
renchérissait, relevant le défi de faire plus fort que le journal. Le roman de Kourouma en dit plus, va plus loin, énonce plus juste. Il y a comme une forfanterie dans son dire, très sensible. Le romancier entend épuiser son objet, comme on le voit à travers le ressassement du discours chez un enfant-soldat dont la naïveté a quelque chose de fascinant. Épargne et dépenses confondues: il s'agit tout ensemble d'accumuler les informations et de les prodiguer dans un gaspillage de phrases et de mots. Avec l'espoir de tout englober en une seule masse, mais avec le sentiment que la progression n'aura pas de fin. À l'image d'un serpent qui se mord la queue, le roman de Kourouma reprend ainsi le motif de la boucle en se terminant sur les mêmes mots que ceux par lesquels il a commencé: "J'ai décidé. Le titre définitif et complet de mon blablabla est: Allah n'est pas obligé d'être juste dans toutes ses choses ici-bas. J'ai commencé à conter mes salades pendant plusieurs jours. » L'écriture démultiplie ses modes d'action. Aspect le plus visible, le roman comble l'espace qui est tout à la fois celui du texte et celui du référent. Il a la réputation de décrire en inventoriant sans retenue, comme en témoigne Bleu blanc rouge d'Alain Mabanckou, qui procède à l'étalage des biens de consommation jusqu'à éprouver la patience de son lecteur:

Ce jour qui me revient à l'esprit, il était vêtu d'un costume sur mesure de Francesco Smalto. Une chemise très transparente laissait deviner sa peau blanchie une fois qu'il avait tombé la veste publiquement. Sa cravate en soie arborait des motifs minuscules de la tour Eiffel. Il ne chaussait que des Weston et était le seul au pays à en posséder en crocodile; le prix d'une paire était l'équivalent du salaire d'un ministre d'État du pays ${ }^{10}$.

Forme d'expansion non moins flagrante, celle qui se joue des proportions narratives. Celle-ci consiste à égarer le texte en des détails et des détours. Le récit surfe sur de petites notations, et c'est comme s'il n'allait pas à l'essentiel. Impression trompeuse évidemment. Le grave finira par se dire, la crise finira par éclater. Mais toujours nous n'y viendrons que par de nombreux biais. Cette technique accompagne avec bonheur ces inactifs intellectuels que sont les héros mudimbiens qui dissertent sur tout. On la retrouve sans surprise dans les romans de Kourouma, qui, empruntant des chemins de traverse, donnent le temps au récit d'accoucher de sa vérité. Elle est même à l'œuvre dans le conte

10. Alain Mabanckou, Bleu blanc rouge, Paris, Présence africaine, 1998, p. 69. 
philosophique de Diop ${ }^{11}$, qui ménage les effets de surprise sous la banalité d'un propos qui s'étire. Mudimbe préludait à ces jeux du visible et du caché dans Entre les eaux et, plus spécialement, dans L'écart, où la narration se perd dans les sables de l'incertitude ou de la velléité.

La digression, ou ce que j'ai appelé ailleurs ${ }^{12}$ la polyfocalisation, est une autre façon pour le texte de sortir de ses limites et de ses normes. Ici, la tendance expansive touche au propos tenu. C'est un peu comme si le récit ne pouvait contrôler ce dernier ou encore comme s'il éprouvait le besoin d'indiquer ses développements possibles. Déjà Sembène, qui laisse le commentaire submerger le récit des faits ${ }^{13}$, est en permanence guetté par cette propension à en rajouter et à digresser. Tous les romans de Kourouma sont portés par une dérive continue du discours et de son propos, même si la narration finit le plus souvent par renouer avec sa ligne principale. Toujours est-il que la polyfocalisation rend compte d'un roman qui s'est développé par dilatations successives. Dans Allah n'est pas obligé, le langage lui-même est pris cette fois dans la spirale inflatoire et se voit emporté dans une exubérance rageuse. Il fait du roman une vaste parlerie. Celle-ci a beau prendre en écharpe maintes informations et éléments de représentation, elle semble se résorber en sa parole bavarde et comme inextinguible. Ce réalisme du mot, de la voix, du ton, parce qu'il met en péril la représentation même à travers ses débordements et parce qu'il figure un discours qui n'a aucune raison de jamais s'arrêter, représente une limite qui ne pourra guère être dépassée.

D'où l'idée de totalisation du roman, qui s'impose à partir de quelques traits distinctifs que je viens d'indiquer: accumulation de l'information, expansion de l'écriture, architecture monumentale, apparente complétude. Pour s'accomplir, toute entreprise de totalisation exige cependant qu'un élément particulier vienne boucler l'ensemble, dans la mesure où elle projette de produire un univers romanesque tellement dense et plein qu'il paraît se refermer sur lui-même et assurer ainsi son autonomie. Shaba deux de Mudimbe est un roman trop calculé pour être expansif comme

11. Boubacar Boris Diop, Le cavalier et son ombre, Paris, Stock, 1997.

12. Justin Bisanswa, "Jeux de miroirs: Kourouma l'interprète", Présence francophone, Sherbrooke, $n^{\circ}$ 59, 2002, p. 8-27.

13. Ousmane Sembène, Les bouts de bois de Dieu, Paris, Présence africaine, 1971. 
d'autres. Mais toutes ses parties se répondent si étroitement, il produit si fortement l'image d'un «tout petit monde» qu'il donne la sensation de son autosuffisance.

Peut-être que Mudimbe aurait voulu écrire un «livre sur rien", selon l'expression de Flaubert à propos de Madame Bovary, et il n'a pas été si loin d'y parvenir. Or, il est permis de se demander si les "livres sur tout» - les romans totaux - ne s'inversent pas aisément en livres sur rien. En attendant le vote des bêtes sauvages ou Monnè, outrages et défis sont des romans très foisonnants, mais dans lesquels rien n'arrive vraiment et où, pour bien peu, le récit s'enlise ou s'égare. Pareillement, ces curieux romans de la quête dans les profondeurs de soi que sont Le chercheur d'Afriques ${ }^{14}$ ou Le lys et le flamboyant ${ }^{15}$ de Lopes sont très chargés et, en même temps, tournent à vide. L'expansion sonyienne du discours va dans le sens d'un rabâchage sans fin et comme dépourvu de nécessité. L'idéal de Mabanckou (dans Bleu blanc rouge), de Biyaoula (dans L'impasse) ou de Beyala est de narrer des destins en rupture, mais qui reviennent au point de départ comme si rien n'avait eu lieu que le lieu. Travaillé ou non par ce paradoxe, le roman africain n'en demeure pas moins très divers dans ses formules et dans ses façons de totaliser. Passons en revue quelquesunes d'entre elles.

\section{Métonymies et allégories}

À cet égard, Mudimbe comme Boris Diop méritent une place particulière. Ils n'ont donné ni dans la dépense scripturale ni dans le grand échafaudage. Leurs romans sont indépendants les uns des autres. Leur style est relativement contenu, aimant à cultiver soit l'allusion, soit l'ellipse. Bref, même si certaine propension au détail est à l'œuvre ici ou là, elle ne verse jamais dans l'expansion sans frein. On a affaire à un réalisme d'une autre nature, sur la qualité duquel je ne reviendrai pas dans ce texte. Mais il est un élément singulier qui fait le lien avec les autres romanciers. Ainsi, d'un texte à l'autre, chacun des romanciers creuse toujours la même problématique. Pierre Landu et Marie-Gertrude, en dépit de la différence de sexe, sont des héros de l'insatisfaction qui poursuivent l'accomplissement de rêves trompeurs dans des

14. Henri Lopes, Le chercheur d'Afriques, Paris, Seuil, 1992.

15. Henri Lopes, Le lys et le flamboyant, Paris, Seuil, 1997. 
expériences décevantes au sein d'un milieu sans horizon. Ces deux religieux se demandent comment un Africain, fidèle à l'Église catholique et donc à la culture occidentale, peut être utile à sa société en ne renonçant pas au message évangélique. Kadidja et le narrateur du Cavalier et son ombre scrutent le problème de la guerre entre ethnies différentes et de l'abus de pouvoir. On ne saurait parler ici ni de série ni de variation sur un thème, mais bien d'une économie de l'approfondissement visant à condenser toute la socialité d'une époque en un scénario très concerté. Renouvelé d'une tentative à l'autre, un seul et même schéma se fait la figure d'une époque, d'un état de société, d'une collectivité.

À la différence des autres romanciers, Mudimbe va donc agir sur et par la rareté du texte. Entendons par là qu'il va élaborer des scénarios qui tendent à ramasser le sens en un nombre restreint de figurations. Deux procédés y sont à l'œuvre. Le premier consiste à déployer la narration selon un éventail fini de possibilités: positions, carrières, amours. Les solutions sont essayées tour à tour. Plus que tout autre, le héros mudimbien est placé face à plusieurs voies ${ }^{16}$ et se demande anxieusement laquelle choisir, quitte à n'en prendre aucune. Le second procédé revient à pratiquer des montages scéniques à forte condensation. Une séquence comme celle de Marie-Gertrude contemplant et analysant la grande reproduction de la Cène d'Andrea del Castagno ${ }^{17}$, une autre comme celle des histoires enchâssées des seigneurs de guerre dans celle de l'enfant-soldat ${ }^{18}$, s'entourent d'une aura d'intensité. Elles sont, à proprement parler, emblématiques. Les deux exemples que je viens de donner dénotent d'ailleurs une volonté de conférer à une scène centrale une fonction de mise en abyme du texte. Ainsi toute une figuration interne se

16. Les titres des romans (Entre les eaux, Le bel immonde, L'écart, Shaba deux) indiquent déjà l'embarras du choix du héros.

17. «Elle orne, tout en long, une grande partie du mur que troue, à l'extrêmedroite, la double porte vitrée d'entrée au réfectoire. Je pense bien connaître ce tableau. Je l'ai, en effet, sous les yeux tous les jours, et cela depuis des années. Mais cette nuit, il m'intriguait. Jean y a la tête proche de la poitrine de Jésus. [...] Pierre, étonné, d'un regard très clair, semble interroger Jésus qui, visiblement, s'attendrit sur l'amour de Jean. En face du Seigneur, Judas. Il a le profil du nez fortement dessiné et semble observer, un sourire imperceptible aux lèvres, mais l'attention vive. Une atmosphère de tension extrême paraît flotter sur ces visages, malgré l'impression générale de communion offerte par le tableau» (Valentin-Yves Mudimbe, Shaba deux. Les carnets de mère MarieGertrude, Paris, Présence Africaine, 1989, p. 30).

18. Dans Kourouma, Allah n'est pas obligé, Paris, Seuil, 2000. 
produit qui est typiquement d'ordre métonymique. La séquence élue parle pour l'ensemble et le résume. On peut donc dire que Mudimbe comme Kourouma ont étendu la métonymie aux dimensions du roman entier. Les vies de Fama, Djigui, Nara, Landu se lisent comme des condensés hautement figuratifs d'une tranche d'Histoire. Elles ne s'y prêtent qu'au prix d'une ironisation de cette Histoire et de toutes les histoires similaires auxquelles cette dernière a donné sens. Mais telle est bien la lecture désabusée que les deux romanciers font de leur temps. Et l'on n'en attendait guère moins de deux romanciers dont le sens de l'humour ne pouvait que prendre acte de l'ironie du destin au moment où bégayait l'Afrique qu'on dit postcoloniale et où elle s'enlisait dans les conventions et l'opportunisme le plus médiocre.

Roman Jakobson a montré que le grand principe métonymique régissait le récit réaliste en général, alors que la métaphore était la figure matricielle du discours poétique ${ }^{19}$. De fait, la métonymie est omniprésente dans les romans africains, et on peut déjà en reconnaître la manifestation à chaque fois que, dans la chaîne causale, une ellipse se rend sensible et que, par exemple, un effet renvoie à une cause informulée. Après tout, c'est par «Elle referma les boutons de sa robe» que Mudimbe nous apprend que Ya et le Ministre viennent de faire l'amour dans le bureau de ce dernier. À tout moment, le roman réaliste est donc susceptible de glisser vers un effet métonymique, du simple fait que, ne pouvant tout dire, il sélectionne dans la chaîne narrative tel énoncé de manière à ce qu'il puisse toujours tenir lieu d'un autre. Mais pour que l'on puisse parler vraiment de métonymie, ce n'est pas suffisant. Il importe encore que le caractère figural, toujours analogique à quelque titre, soit rendu sensible par une marque ou par une autre.

Sans produire de métonymies rhétoriquement qualifiées, la plupart des romans africains, réalistes, baignent dans ce que l'on pourrait appeler un climat métonymique général. Dans Monnè, outrages et défis, Kourouma raconte la geste coloniale française en Afrique occidentale. À travers la métaphore filée du train, nous assistons donc à l'ascension sociale et aux déboires du roi Djigui. Cette montée prendra du temps et connaîtra des obstacles.

19. Voir notamment «Deux aspects du langage et deux types d'aphasie», dans Roman Jakobson, Essais de linguistique générale, traduit de l'anglais par Nicolas Ruwet, Paris, Minuit, coll. «Points», 1963, p. 43-67. 
Irrésistible pourtant, aux yeux des observateurs, elle apparaîtra au lecteur comme vocation à devenir ce que l'on est. Très significative, à cet égard, la quasi-dévotion que le peuple voue à son roi :

Oui, Djigui était un chemin récurrent, quand on le terminait et arrivait au sommet, il se déployait, s'éloignait, et il fallait le recommencer comme si on n'en avait rien dit. Allah lui avait conféré ce qu'Il a donné à peu de prophètes: à la fois la fortune, la longévité et la nombreuse progéniture. Cela n’avait pas suffi: Il lui avait accordé encore d'être le patriarche le plus sorcier, féticheur et musulman du pays, le plus méchant et charitable, le plus aimé et haï. Il reste dans le pays l'objet de chaudes vénérations et, avec les indépendances, certains démagogues pour faire passer quelques contradictions et retarder de quelques jours le coup d'État inexorable qui, lorsqu'il ne les tue pas, les envoie à la retraite anticipée en Suisse ou en France, se réclament de lui, se l'approprient, le citent à toute occasion et effectuent de fréquents pèlerinages sur sa tombe ${ }^{20}$.

Reflet, effet: l'image fixe très tôt le héros dans le rôle - micandide mi-cynique - dans lequel il ne fera que se complaire et dans lequel ses partenaires s'attacheront à le confirmer. Comme si l'effet était partout et la cause nulle part. À se généraliser ainsi, la métonymie perd beaucoup de son identité. On se trouve en présence d'une disposition narrative qui a pour effet de noyer la figure dans l'indistinction. Les occurrences multiples du dispositif finissent d'ailleurs par se résorber dans de grandes entités aussi floues que la déchéance ou la misère, comme dans l'exemple qui suit, tiré de Mudimbe:

Pensez: en dix ans d'indépendance, le taux de scolarisation est tombé de trente-sept points, le pouvoir d'achat a baissé de cinq cents points, le nombre de chômeurs a augmenté de deux millions, le taux de mortalité grimpé de vingt-sept pour cent. Cinq dévaluations, une tous les deux ans. Ah, les nègres... Heureusement, il y a la religion pour le bon peuple... Commode n'est-ce pas? Le miracle permanent... Contre tous les maux... On en oublie le nombre toujours croissant de gosses qui crèvent de variole ou de rougeole ${ }^{21}$.

Dans Bleu blanc rouge, la métonymie donne toute sa mesure, apparaissant sous des espèces fort variées. À croire que l'écrivain a voulu proposer dans son roman une grammaire complète du

20. Ahmadou Kourouma, Monnè, outrages et défis, Paris, Seuil, 1990, p. 284.

21. Valentin-Yves Mudimbe, L'écart, Paris, Présence africaine, 1976, p. 92. 
procédé. Le principe ne varie guère: la description porte à l'avantplan un objet pittoresque qu'elle détaille; il est apparent que, ainsi exhibée, la chose est figure de celui qui la détient et image de tout son être social. C'est ainsi que Mabanckou affuble Moki d'objets kitsch qui, dans leur bêtise, ont un grand pouvoir allusif. Chaque fois, la chose, présentée en pièce montée, se nimbe d'un grotesque qui rejaillit sur tout le contexte. On remarquera que, au gré d'une vision tronquée (discours croisés et fragmentés des candidats à l'immigration à Paris), un contenant est donné pour son contenu. Façon, pour l'écrivain, de faire ressortir l'absurdité des situations, mais surtout usage ironique de la métonymie qui, face à l'obscénité des choses, devient litote en disant le moins pour dire le plus.

Métonymie d'art que celle de Mudimbe ou de Kourouma, mais qui travaille le sens au plus près. Substituant à chaque occasion l'objet à l'être, elle reflète un univers où valeurs et sentiments sont, à proprement parler, réifiés. Il faut bien voir qu'à tant commettre d'erreurs d'appréciation sur la vie et sur les êtres, Fama et Djigui s'épuisent en un vain combat. De même, ils sont eux-mêmes la proie des choses, se révélant les premiers consommateurs de la modernité occidentale, avide d'objets en toc et de technologie (la métaphore du train) à la mode. Les pauvres Fama et Djigui sont si bien de leur époque qu'ils en sont, à eux seuls, la métonymie.

Par la vertu métonymique, chaque grand personnage est non seulement de son temps, mais est son temps. Sony Labou Tansi le sait, qui baptise son personnage le Guide providentiel ou Son Excellence. Fama aussi est une époque, au point d'incarner, au sommet de sa déchéance, la fin de la dynastie et la nostalgie du bon vieux temps. Et Djigui illustre un monde dynastique, perdu dans la modernité. Associations par le haut que contrebalancent d'innombrables associations par le bas. Que de personnages inséparables d'une singularité physique, d'un objet, d'une formule, d'un emblème: Salimata et sa stérilité, Penda et ses haillons de menstrues pendant la grève de Dakar-Niger, Ya et la boîte de nuit, les mendiants de la ville et le tirage de la «tontine», la mère de Birahima et sa jambe droite pourrie par l'ulcère. Ainsi va la métonymie, qui assure la circulation des signifiés et de leurs connotations au sein d'univers clos et en postulant leur solidarité, c'est-à-dire leur caractère interchangeable.

Les séquences condensatrices que j'ai relevées chez Mudimbe et Kourouma méritent d'être observées plus avant. Elles partagent 
plusieurs traits significatifs: leur position centrale dans le texte; le nœud narratif qu'elles forment; l'étrangeté qui émane d'elles (de ce point de vue, la scène de l'éveil de Marie-Gertrude est plus typique que celle des histoires enchâssées des seigneurs de guerre chez Kourouma: elles vont d'ailleurs ensemble); et une discrète portée symbolique (naissant du contraste entre l'apparence de communion dans le couvent et la tension des visages sur le tableau chez Mudimbe, entre grandeur passée et décadence présente ou splendeur passée et misère présente chez Kourouma). L'aura dont elles sont nimbées incite à voir en elles des allégories au sens moderne que peut prendre le $\operatorname{mot}^{22}$. Il s'agit bien, comme on voit, de séquences participant pleinement du vraisemblable réaliste, mais que leur relative incongruité dote d'un pouvoir figuratif certain. De plus, le montage dont elles sont l'objet en fait des lieux de cristallisation du sens. Tout veut donc que la lecture s'arrête à elles et s'interroge sur leur valeur.

L'allégorie romanesque serait, en somme, une image forte qui, se détachant du contenu narratif, rend sensible mouvement du sens et travail de l'imaginaire. Flirt d'une religieuse en surplomb du sommeil, ou monologue dans son sommeil, tout en appelle, par-delà le drame, à une rêverie où le piment de l'humour et de l'ironie joue son rôle. Les deux situations sont pour le moins incongrues et questionnent un contexte dominé par les exigences les plus strictes de la normalité. Dès lors, elles ne peuvent que sursignifier et voir refluer vers elles bien des questions du texte. Condensatrices, elles invitent à une interprétation symbolique, mais de caractère mobile, au sens où le symbole ne peut, en aucune façon, y prendre une forme figée.

En chaque grand roman africain, il est sans doute des séquences allégoriques de ce genre. Elles prennent souvent l'aspect d'une petite scène dans laquelle un destin se résume et se joue. Elles se trouvent, de ce fait, en situation de rayonner sur l'ensemble du texte et de prendre valeur emblématique par rapport à lui. Notons, cependant, que leur valeur symbolique incertaine ne facilite pas toujours le repérage. Et, dès lors, leur existence est

22. C'est notamment à partir des écrits du philosophe allemand Walter Benjamin que cette notion d'allégorie s'est vue remise à l'honneur. Mais, chez Benjamin, l'allégorie prend un sens trop particulier pour que je fasse ici état de cette conception. Voir, à ce propos, Henri Meschonnic, «L'allégorie chez Walter Benjamin, une aventure juive», dans Heinz Wismann (sous la dir. de), Walter Benjamin et Paris, Paris, Cerf, coll. « Passages», 1986, p. 707-741. 
toujours laissée, dans une certaine mesure, à l'appréciation du lecteur ou du critique. Autrement dit, elles sollicitent le travail interprétatif et, éventuellement, le facilitent. Il est sans doute des moyens de les repérer qui peuvent tenir à leur thématique interne. Ainsi de ces séquences où le personnage principal, seul ou avec d'autres, est engagé dans un déplacement ou un voyage qui marque particulièrement son destin. Les personnages des romans africains se déplacent constamment. Dans tous les cas, le voyage prend une valeur initiatique qui dépasse la simple circonstance.

Mais les séquences narratives ne sont pas les seules unités textuelles qui puissent accéder à cette dimension allégorique. On pense, par exemple, à ces motifs récurrents que Mikhail Bakhtine a nommés "chronotopes» et dont il a soutenu qu'ils se retrouvaient dans la plupart des grands corpus romanesques. Le critique considérait, en effet, que chaque romancier possédait ses figures de localisation favorites, et qu'un temps et un espace venaient s'y fondre pour accéder à une signification supérieure. Il précisait: "Ici, le temps se condense, devient compact, visible pour l'art, tandis que l'espace s'intensifie, s'engouffre dans le mouvement du temps, du sujet, de l'Histoire ${ }^{23}$ ». Plus concrètement, il s'agit, dans un corpus romanesque donné, de sites de prédilection où s'exprime un rapport singulier au temps et à l'existence. Chronotope du village ou du «bolloda» chez Kourouma où le héros se reprend et adhère à lui-même; chronotope du jardin, du maquis, de la bibliothèque, du couvent, des boîtes de nuit chez Mudimbe, où se déroulent de manière feutrée les batailles sociales; chronotope de la forêt, de la chambre d'hôtel et du palais chez Sony, où la durée se fige à l'écluse pour donner le temps au personnage de se découvrir. On voit combien ces lieux marqués détiennent un intense pouvoir au sein du récit, condensateur et médiateur tout à la fois. Le sens de l'action s'y ramasse pour ouvrir à d'autres valeurs. Avec ces derniers exemples, on serait même enclin à croire que l'allégorie voue le roman à l'implosion. C'est plutôt son pouvoir structurant qui prévaut. Figure de convergence et de scansion, l'allégorie offre au récit des points d'appui, des lieux de fixation. Façon éminente d'endiguer par un effet transcendant la dispersion ou l'inflation qui, je l'ai montré, toujours guette ou, encore, de rejoindre l'ambition totalisante que nous savons.

23. Mikhaïl Bakhtine, Esthétique et théorie du roman, Paris, Gallimard, 1978, p. 237. 


\section{Conclusion}

Il n'est pas de roman sans imaginaire. Il n'est pas non plus d'écriture sans folie. Le roman africain ne fait pas exception, en dépit de ce que certains critiques pouvaient croire. Il délire et extravague, même s'il le fait plus volontiers par à-coups que de manière soutenue. Il est d'ailleurs frappant que les romans les plus aboutis sont aussi ceux qui ouvrent en eux des espaces où certaine «folie» a cours. Folie de forme et folie de contenu, sans distinction nette en bien des cas. Ainsi, par tout un côté, Le lys et le flamboyant est un grand opéra plein d'artifices inspirés. L'écart se lit comme un scénario halluciné traversé d'étonnantes visions. La plupart des romans de Beyala se présentent comme une douloureuse et charmante fantaisie érotique. Les romans de Sony se déroulent en crescendo dans une démesure et une invention burlesque qui paraissent sans frein. Le cavalier et son ombre se donne des airs d'allégorie kafkaïenne. Bref, la supériorité de ces fictions est d'assurer la jonction et l'interaction réciproque entre une analyse exigeante de la socialité et les sollicitations actives d'un imaginaire.

Si l'on voulait dégager des tendances plus générales, on invoquerait à nouveau ces basculements des œuvres hors des limites du contrat de lecture initial. Sony ou Kourouma fantastiques, Mudimbe poétique, Lopes burlesque. En chaque cas, la part est faite à une certaine déraison. Mais il est évidemment plus intéressant de reconnaître cette folie-là au cœur même du projet romanesque africain que dans certains dépassements ou déplacements textuels. À ce moment, la dérive ne porte pas d'étiquette particulière et se trouve générée par le principe conducteur. Sans revenir sur la question de l'imaginaire, dont il y aurait encore beaucoup à dire, considérons qu'une certaine libération pulsionnelle se joue sur les terrains si conjoints du récit et de l'écriture. C'est de folie légère que je parle ici, on s'en doute bien, mais celleci est d'autant plus efficiente qu'elle reste, en quelque sorte, allusive. 